\section{The Eagle jugular syndrome}

\begin{abstract}
Alba Scerrati, ${ }^{1}$ Paolo Zamboni, ${ }^{2}$ Erica Menegatti, ${ }^{2}$ Roberto Galeotti, ${ }^{3}$ Marcello Lapparelli, ${ }^{1}$ Luca Traina, ${ }^{4}$ Mirko Tessari, ${ }^{2}$ Andrea Ciorba, ${ }^{5}$ Pasquale De Bonis, ${ }^{1}$ Stefano Pelucchi ${ }^{5}$

${ }^{1}$ Neurosurgery Unit, Ferrara University Hospital; ${ }^{2}$ Vascular Diseases Center, Ferrara University Hospital; ${ }^{3}$ Interventional Radiology Unit, Ferrara University Hospital; ${ }^{4}$ Vascular Surgery Unit, Ferrara University Hospital; ${ }^{5}$ ENT Unit, Ferrara University Hospital, Ferrara, Italy
\end{abstract}

\section{Background}

The elongation of the styloid process is historically associated with two variants of the Eagle syndrome. The classic one, mainly characterized by pain and dysphagia, and the carotid variant characterized by pain and sometimes by cerebral ischemia. We observed a further variant characterized by a styloid elongation coursing adjacent to the transverse process of $\mathrm{C} 1$ (Figure 1), causing significant compression of the internal jugular vein. ${ }^{1-4}$

\section{Methods}

We revised all the cases of Eagle syn- drome, including the jugular variant, admitted in our Hospital in the last six years. We compared symptomatology, associated comorbidities and imaging. Data were statistically analyzed.

\section{Results}

Overall 23 patients were admitted to the Hospital for symptomatic elongation of the styloid process, 11 male and 12 females. The jugular variant of the Eagle syndrome (Figure 1) is clinically delineated by significant differences, as compared with the classic variant and carotid variants. Headache was the more prominent symptom $(\mathrm{P}<0.009)$ as well as a documented peri-mesencephalic hemorrhage was the more significant comorbidity $(\mathrm{P}<0.0003)$. The group classic-carotid variant was characterized by ipsilateral pain compared to the jugular variant $(\mathrm{P}<0.0003)$. $\mathrm{CT}$ angiography with venous phase extended to the neck veins and imaging reconstruction is highly recommended as imaging technique, complemented by color-Doppler ultrasound.

\section{Conclusions}

The elongation of the styloid process may have different paths, which creates compression on the surrounding anatomical structures. There may be a possible association of jugular impingement by an elongated styloid process with symptoms.

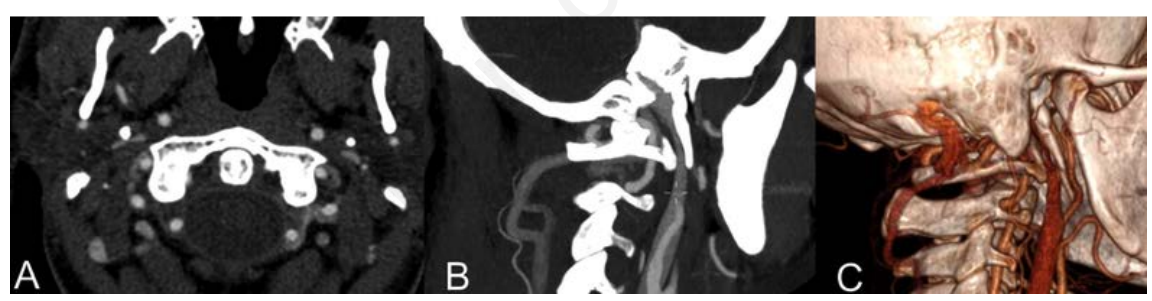

\title{
References
}

1. Jayaraman MV, Boxerman JL, Davis LM, et al. Incidence of extrinsic compression of the internal jugular vein in unselected patients undergoing CT angiography. AJNR Am J Neuroradiol 2012;33:1247-50.

2. Zamboni P. How to objectively assess jugular primary venous obstruction. Veins and Lymphatics 2014;3:4195.

3. Ho S, Luginbuhl A, Finden S, et al. Styloid/C1 transverse process juxtaposition as a cause of Eagle's syndrome. Head Neck 2015;37:E153-6.

4. Dashti SR, Nakaji P, Hu YC, et al. Styloidogenic jugular venous compression syndrome: diagnosis and treatment: case report. Neurosurgery 2012;70:E795-9.

Figure 1. CT angio of the illustrative case showing a right jugular internal vein bone nutcracker between the $\mathrm{C} 1$ transverse process and the elongated styloid process. A) Axial cut; B) Longitudinal reconstruction; C) 3D reconstruction.
Correspondence: Alba Scerrati, Neurosurgery Italy.

Conference presentation: $9^{\text {th }}$ Annual Meeting
of the International Society of Neurovascular Disease (ISNVD), May $30^{\text {th }}-31^{\text {st }}, 2019$, Award: Young Investigator Award Session This work is licensed under a Creative (Copyright: the Author(s), 2019

Veins and Lymphatics 2019; 8:8441 doi: $10.4081 / v l .2019 .8441$ 